Internat. J. Math. \& Math. Sci.

Vol. 24, No. 6 (2000) 403-418

S0161171200002180

(C) Hindawi Publishing Corp.

\title{
A COMMUTATOR THEOREM FOR FRACTIONAL INTEGRALS IN SPACES OF HOMOGENEOUS TYPE
}

\author{
JORGE J. BETANCOR
}

(Received 22 October 1998)

\begin{abstract}
We give a new proof of a commutator theorem for fractional integrals in spaces of homogeneous type.
\end{abstract}

Keywords and phrases. Commutator fractional integrals, homogeneous type spaces.

2000 Mathematics Subject Classification. Primary 42B20.

1. Introduction. Bramanti and Cerutti [3] and Bramanti [2] extended a classical commutator theorem for fractional integrals due to Chanillo [5] to the context of spaces of homogeneous type. In [3] Bramanti and Cerutti follow an idea contained in [7], based in holomorphic families of operators, used to study the $L^{p}$ boundedness of singular integrals in Euclidean spaces. In [2] Bramanti investigated the boundedness of the commutator of certain integral operators having positive kernels. A fractional integral appears as a particular case. Bramanti deduces the boundedness of the commutator from a suitable inequality that involves the maximal sharp function. In this paper, we give a different proof to the commutator theorem for fractional integrals in spaces of homogeneous type. We follow the original proof of Chanillo [5] and a good $\lambda$ inequality is essential.

We firstly recall the main definitions needed in the paper (see $[8,9,11]) .(X, \delta, \mu)$ will be a space of homogeneous type. That is, $X$ is a nonvoid set, $\delta$ is a quasidistance on $X$, i.e., $\delta: X \times X \rightarrow[0, \infty)$ is a function satisfying the following properties:

(i) $\delta(x, y)=0$ if and only if $x=y$,

(ii) $\delta(x, y)=\delta(y, x)$, for every $x, y \in X$, and

(iii) there exists a positive constant $k$ such that for every $x, y, z \in X$

$$
\delta(x, y) \leq k(\delta(x, z)+\delta(z, y)),
$$

and $\mu$ is a positive regular measure on $X$ defined on a $\sigma$-algebra of subsets of $X$ which contains the open sets (in the topology induced by the uniform structure associated to $\delta$ ) and the ball $B(x, r)=\{y \in X: \delta(x, y)<r\}$, for every $x \in X$ and $r>0$, and that satisfies the doubling condition: there exists $A>0$ for which

$$
0<\mu(B(x, 2 r)) \leq A \mu(B(x, r)),
$$

for each $x \in X$ and $r>0$. Note that if $X$ has more than one element, then $k \geq 1$. The trivial case $k<1$ is not considered in this paper.

There are many interesting examples of spaces of homogeneous type. For instance, any $C^{\infty}$ compact Riemannian manifold with the Riemaniann metric and volume and 
the boundary of any bounded Lipschitz domain in $\mathbb{R}^{n}$ with the induced Euclidean metric and the Lebesgue measure are spaces of homogeneous type.

A space of homogeneous type is said to be normal if there exist positive constants $A_{1}$ and $A_{2}$ such that for every $x \in X$,

$$
\begin{gathered}
A_{1} r \leq \mu(B(x, r)), \quad \text { when } 0<r<R_{x}, \\
\mu(B(x, r)) \leq A_{2} r, \quad \text { if } r \geq r_{x},
\end{gathered}
$$

where

$$
\begin{aligned}
& R_{x}= \begin{cases}\infty, & \text { if } \mu(X)=\infty, \\
\inf \{r>0: B(x, r)=X\}, & \text { if } \mu(X)<\infty,\end{cases} \\
& r_{x}= \begin{cases}0, & \text { if } \mu(\{x\})=0, \\
\sup \{r>0: B(x, r)=\{x\}\}, & \text { if } \mu(\{x\})>0 .\end{cases}
\end{aligned}
$$

Sufficient conditions, in order that a space $(X, \delta, \mu)$ of homogeneous type admits a quasidistance $d$ that is equivalent to $\delta$ and such that $(X, d, \mu)$ is normal, are given in [14, Lemma 22].

A space of homogeneous type is of order $\rho, 0<\rho \leq 1$, if there is a positive constant $C$ such that for every $x, y, z \in X$

$$
|\delta(x, z)-\delta(y, z)| \leq C \delta(x, y)^{\rho}(\max \{\delta(x, z), \delta(y, z)\})^{1-\rho} .
$$

For each $1 \leq p \leq \infty, L^{p}(X, \mu)$ and $\|\cdot\|_{p}$ have the usual meanings. We say that a complex valued measurable function $f$ on $X$ is in $L_{\mathrm{loc}}^{p}(X, \mu), 1 \leq p<\infty$, if $\int_{B(x, r)}|f(x)|^{p} d \mu(x)<$ $\infty$, for every $r>0$ and for some (and then for all) $x \in X$.

Let $b \in L_{\text {loc }}^{1}(X, \mu)$. We define $b_{\epsilon}(x)$, with $x \in X$ and $\epsilon>0$, as the mean value

$$
b_{\epsilon}(x)=\frac{1}{\mu(B(x, \epsilon))} \int_{B(x, \epsilon)} b(y) d \mu(y) .
$$

If $1 \leq p<\infty$ we will say that a function $b \in L_{\mathrm{loc}}^{p}(X, \mu)$ is in $B M O_{p}$ if and only if,

$$
\|b\|_{*, p}=:\left\|\sup _{\epsilon>0}\left\{\frac{1}{\mu(B(x, \epsilon))} \int_{B(x, \epsilon)}\left|b(y)-b_{\epsilon}(x)\right|^{p} d \mu(y)\right\}^{1 / p}\right\|_{\infty}<\infty .
$$

We define on $B M O_{p}$ a "norm" as follows:

$$
\|b\|^{(p)}= \begin{cases}\|b\|_{*, p}, & \text { if } \mu(X)=\infty, \\ \|b\|_{*, p}+\left|\int_{X} b(x) d \mu(x)\right|, & \text { if } \mu(X)<\infty .\end{cases}
$$

When $\mu(X)<\infty,\left(B M O_{p},\|\cdot\|^{(p)}\right)$ is a Banach space. If $\mu(X)=\infty$, then we introduce in $B M O_{p}$ the following relation: let $b_{1}$ and $b_{2}$ be in $B M O_{p}$,

$$
b_{1} \sim b_{2} \Longleftrightarrow \text { there exists } C \in \mathbb{C} \text { such that } b_{1}-b_{2}=C \text {. }
$$

It is clear that if $b_{1}, b_{2} \in B M O_{p}$ and $b_{1} \sim b_{2}$, then $\left\|b_{1}\right\|^{(p)}=\left\|b_{2}\right\|^{(p)}$. The quotient space $B M O_{p} / \sim$ will be denoted again by $B M O_{p}$ and by considering on it the norm 
induced by $\|\cdot\|^{(p)}, B M O_{p}$ is a Banach space. As it was proved by Coifman and Weiss [9, page 594], if $1 \leq p, q<\infty$, the spaces $B M O_{p}$ and $B M O_{q}$ coincide and the norms $\|\cdot\|^{(p)}$ and $\|\cdot\|^{(q)}$ are equivalent. In the sequel, as usual, we will denote by BMO the space $B M O_{p}, 1 \leq p<\infty$.

Let $0 \leq \alpha<1$. The fractional maximal function $M_{\alpha} f$ of $f \in L_{\mathrm{loc}}^{1}(X, \mu)$ is defined by

$$
\left(M_{\alpha} f\right)(x)=\sup _{B: x \in B} \frac{1}{\mu(B)^{1-\alpha}} \int_{B}|f(y)| d \mu(y), \quad x \in X .
$$

Here, for each $x \in X$, the supremum is taken over all those $B$ balls in $X$ containing to $x$. As usual we denote by $M$ the maximal operator $M_{0}$.

The fractional integral of order $\alpha$ of $f, I_{\alpha} f$, is given by

$$
\left(I_{\alpha} f\right)(x)=\int_{X-\{x\}} \frac{f(y)}{\delta(x, y)^{1-\alpha}} d \mu(y) .
$$

In this paper, we study the boundedness of the commutator $\left[I_{\alpha}, b\right]$ of the fractional integral $I_{\alpha}$ and the multiplier operator associated to a measurable function $b$ on $X$ defined through

$$
\left[I_{\alpha}, b\right](f)=b I_{\alpha}(f)-I_{\alpha}(b f) .
$$

Throughout this paper, for every $1 \leq p<\infty$, we will denote by $p^{\prime}$ the conjugate of $p$. By $C$ we will always represent a positive constant not necessarily the same in each occurrence.

The following theorem is the main result of the paper.

TheOrem 1.1. Let $0<\alpha<1,0 \leq \rho<1,1<p<1 / \alpha$, and $1 / q=1 / p-\alpha$. Assume that $(X, \delta, \mu)$ is a normal space of homogeneous type of order $\rho$ such that $\mu(\{x\})=$ $0, x \in X$. Then the commutator operator $\left[I_{\alpha}, b\right]$ is bounded from $L^{p}(X, \mu)$ into $L^{q}(X, \mu)$ provided that $b \in B M O$.

Let now $(X, \delta, \mu)$ be a normal space of homogeneous type and of order $\rho \in(0,1)$, such that $\mu(X)=\infty$ and $\mu(\{x\})=0$, for every $x \in X$. Gatto, Segovia, and Vagi [10] defined, for every $0<\alpha<1$, a function $\delta_{\alpha}$ on $X \times X$ as follows:

$$
\delta_{\alpha}(x, y)=\left(\int_{0}^{\infty} t^{\alpha-1} s(x, y, t) d t\right)^{1 / \alpha-1}, \text { for } x \neq y,
$$

where $s$ represents a symmetric approximation to the identity in the sense of Coifman, and

$$
\delta_{\alpha}(x, y)=0, \text { for } x=y .
$$

In [10, Lemma 2] it is proved that, for every $0<\alpha<1, \delta_{\alpha}$ is a quasidistance equivalent to $\delta$. Moreover, for each $0<\alpha<1,\left(X, \delta_{\alpha}, \mu\right)$ is a normal space of homogeneous type of order $\rho$.

Also these authors introduced the fractional integral $\tilde{I}_{\alpha}$ of order $\alpha \in(0,1)$ through

$$
\left(\tilde{I}_{\alpha} f\right)(x)=\int_{X-\{x\}} \frac{f(y)}{\delta_{\alpha}(x, y)^{1-\alpha}} d \mu(y) .
$$


If we represent by $B M O(\alpha)$ the $B M O$-space associated to the quasidistance $\delta_{\alpha}, 0<\alpha<$ 1 , it is immediately deduced from Theorem 1.1 the following commutator theorem for the fractional integral $\tilde{I}_{\alpha}$.

COROLlaRY 1.2. Assume that $(X, \delta, \mu)$ is a normal space of homogeneous type and of order $\rho \in(0,1)$, such that $\mu(X)=\infty$ and $\mu(\{x\})=0$, for every $x \in X$. Let $0<\alpha<1$. Then the commutator operator $\left[\tilde{I}_{\alpha}, b\right]$ defined by

$$
\left[\tilde{I}_{\alpha}, b\right](f)=b \tilde{I}_{\alpha}(f)-\tilde{I}_{\alpha}(b f),
$$

is a bounded operator from $L^{p}(X, \mu)$ into $L^{q}(X, \mu)$ provided that $1<p<1 / \alpha, 1 / q=$ $1 / p-\alpha$ and $b \in B M O(\alpha)$.

2. The proof of the commutator theorem. In this section, we will prove Theorem 1.1. To see that result we previously establish six lemmas.

Boundedness of the fractional integral $I_{\alpha}$ was studied in [11, Theorem 1] and [12, Theorems 2.2 and 2.4].

LEMmA 2.1 (see [11, Theorem 1]). Let $1<p<1 / \alpha$ and $1 / q=1 / p-\alpha$. If $(X, \delta, \mu)$ is a normal space of homogeneous type, then

(i) $I_{\alpha}$ maps continuously $L^{p}(X, \mu)$ into $L^{q}(X, \mu)$.

(ii) There exists $C_{1}>0$ such that

$$
\mu\left(\left\{x \in X:\left|I_{\alpha}(f)(x)\right|>\lambda\right\}\right) \leq C_{1}\left(\frac{\|f\|_{1}}{\lambda}\right)^{1 / 1-\alpha},
$$

for every $f \in L^{1}(X, \mu)$ and $\lambda>0$.

Kokilashvili and Kufner [12, Theorem 3.2] proved a weighted version of [11, Theorem 1].

Kokilashvili and Kufner [12] established weighted inequalities for the maximal fractional operator $M_{\alpha}$. Also Wheeden [15] and Bernardis and Salinas [1] gave characterizations for the pairs of weight functions for which $M_{\alpha}$ is a bounded operator between the corresponding weighted $L^{p}$-spaces.

The following result can be easily infered from [15, Theorem 4] (also from [12, Proposition $\mathrm{A}]$ ).

LEMMA 2.2. Let $1<p<1 / \alpha$ and $1 / q=1 / p-\alpha$. Then $M_{\alpha}$ is a bounded operator from $L^{p}(X, \mu)$ into $L^{q}(X, \mu)$.

We now define the auxiliar operator $C(b, f)$ on $X$ as follows:

$$
C(b, f)(x)=\sup _{\epsilon>0}\left|\int_{X \backslash B(x, \epsilon)} \frac{b(y)-b_{\epsilon}(x)}{\delta(x, y)^{1-\alpha}} f(y) d \mu(y)\right|, \quad x \in X,
$$

where $b$ and $f$ are measurable complex functions on $X$.

Next a useful weak type inequality for the operator $C(b, f)$ is established. 
LEMmA 2.3. Assume that $(X, \delta, \mu)$ is a normal space of homogeneous type. Let $1<$ $p<1 / \alpha$. If $f \in L^{p}(X, \mu)$ and $b \in L^{p^{\prime}}(X, \mu)$, then

$$
\mu(\{x \in X: C(b, f)(x)>\lambda\}) \leq C_{0}\left(\frac{\|b\|_{p^{\prime}}\|f\|_{p}}{\lambda}\right)^{1 / 1-\alpha}, \quad \text { for every } \lambda>0 .
$$

Proof. It is not hard to see that

$$
\begin{aligned}
C(b, f)(x) \leq & \sup _{\epsilon>0} \int_{X \backslash B(x, \epsilon)} \frac{|b(y)||f(y)|}{\delta(x, y)^{1-\alpha}} d \mu(y) \\
& +\sup _{\epsilon>0}\left|b_{\epsilon}(x)\right| \int_{X \backslash B(x, \epsilon)} \frac{|f(y)|}{\delta(x, y)^{1-\alpha}} d \mu(y) \\
\leq & I_{\alpha}(|b f|)(x)+I_{\alpha}(|f|)(x) M(b)(x), \quad x \in X .
\end{aligned}
$$

Moreover Holder inequality and Lemmas 2.1 and 2.2 lead to

$$
\begin{aligned}
& \int_{X} M(b)(x)^{1 / 1-\alpha} I_{\alpha}(|f|)(x)^{1 / 1-\alpha} d \mu(x) \\
& \quad \leq\left(\int_{X} M(b)(x)^{p^{\prime}} d \mu(x)\right)^{1 / p^{\prime}(1-\alpha)}\left(\int_{X} I_{\alpha}(|f|)(x)^{p^{\prime} / p^{\prime}(1-\alpha)-1} d \mu(x)\right)^{1-\left(1 / p^{\prime}(1-\alpha)\right)} \\
& \quad \leq C\|b\|_{p^{\prime}}^{1 / 1-\alpha}\|f\|_{p}^{1 / 1-\alpha} .
\end{aligned}
$$

Hence if $\lambda>0$, then

$$
\mu\left(\left\{x \in X: M(b)(x) I_{\alpha}(|f|)(x)>\lambda\right\}\right) \leq C\left(\frac{\|b\|_{p^{\prime}}\|f\|_{p}}{\lambda}\right)^{1 / 1-\alpha} .
$$

Also by taking into account Lemma 2.1 we have

$$
\begin{aligned}
\mu\left(\left\{x \in X: I_{\alpha}(|b f|)(x)>\lambda\right\}\right) & \leq C\left(\frac{\|b f\|_{1}}{\lambda}\right)^{1 / 1-\alpha} \\
& \leq C\left(\frac{\|b\|_{p^{\prime}}\|f\|_{p}}{\lambda}\right)^{1 / 1-\alpha}, \quad \lambda>0 .
\end{aligned}
$$

Now to finish the proof of this lemma it is sufficient to combine (2.4), (2.6), and (2.7).

LEMMA 2.4. Assume that $(X, \delta, \mu)$ is a normal space of homogeneous type such that $\mu(\{x\})=0, x \in X$. Let $0<\alpha<1,1<p<1 / \alpha, 0<\beta<1 / k$, and $d, \gamma>0$. Let $b \in B M O$ and $f$ be a measurable function. Then

$$
d^{\gamma} \int_{X \backslash B(x, d)} \frac{\left|b(y)-b_{d}(x)\right|}{\delta(x, y)^{1+\gamma-\alpha}}|f(y)| d \mu(y) \leq C\left(M_{\alpha p}\left(|f|^{p}\right)\left(x_{0}\right)\right)^{1 / p}\|b\|_{*, p^{\prime}},
$$

provided that $\delta\left(x, x_{0}\right) \leq \beta d$. Here $C$ is a constant that does not depend on $d$. 
Proof. Suppose that $\mu(X)=\infty$. If $\mu(X)<\infty$ we can proceed in a similar way. Holder inequality implies that

$$
\begin{aligned}
& d^{\gamma} \int_{X \backslash B(x, d)} \frac{\left|b(y)-b_{d}(x)\right|}{\delta(x, y)^{1+\gamma-\alpha}}|f(y)| d \mu(y) \\
& \leq\left(d^{\gamma} \int_{X \backslash B(x, d)} \frac{\left|b(y)-b_{d}(x)\right|^{p^{\prime}}}{\delta(x, y)^{1+\gamma}} d \mu(y)\right)^{1 / p^{\prime}} \\
& \times\left(d^{\gamma} \int_{X \backslash B(x, d)} \frac{|f(y)|^{p}}{\delta(x, y)^{1+\gamma-p \alpha}} d \mu(y)\right)^{1 / p} .
\end{aligned}
$$

Since $\mu$ is doubling we can write for every $x \in X$ and $j \in \mathbb{N}$,

$$
\begin{aligned}
\left|b_{2^{j-1} d}(x)-b_{2^{j} d}(x)\right| & \leq \frac{1}{\mu\left(B\left(x, 2^{j-1} d\right)\right)} \int_{B\left(x, 2^{j-1} d\right)}\left|b(y)-b_{2^{j} d}(x)\right| d \mu(y) \\
& \leq C \frac{1}{\mu\left(B\left(x, 2^{j} d\right)\right)} \int_{B\left(x, 2^{j} d\right)}\left|b(y)-b_{2^{j} d}(x)\right| d \mu(y) \\
& \leq C\|b\|_{*, 1} .
\end{aligned}
$$

Hence, it concludes that

$$
\left|b_{d}(x)-b_{2^{j} d}(x)\right| \leq C j\|b\|_{*, 1}, \quad j \in \mathbb{N}, x \in X .
$$

Then, since $(X, \delta, \mu)$ is normal, it follows

$$
\begin{aligned}
& d^{\gamma} \int_{X \backslash B(x, d)} \frac{\left|b(y)-b_{d}(x)\right|^{p^{\prime}}}{\delta(x, y)^{1+\gamma}} d \mu(y) \\
& \leq d^{\gamma} \sum_{j=0}^{\infty} \int_{2^{j+1} d>\delta(x, y) \geq 2^{j} d} \frac{\left|b(y)-b_{d}(x)\right|^{p^{\prime}}}{\delta(x, y)^{1+\gamma}} d \mu(y) \\
& \leq C d^{\gamma} \sum_{j=0}^{\infty}\left(2^{j} d\right)^{-1-\gamma} \int_{2^{j+1} d>\delta(x, y) \geq 2^{j} d}\left|b(y)-b_{d}(x)\right|^{p^{\prime}} d \mu(y) \\
& \leq C \sum_{j=0}^{\infty} \frac{2^{-j \gamma}}{2^{j} d}\left(\int_{B\left(x, 2^{j+1} d\right)}\left|b(y)-b_{2^{j+1} d}(x)\right|^{p^{\prime}} d \mu(y)\right. \\
& \left.\quad+\left((j+1)\|b\|_{*, 1}\right)^{p^{\prime}} \mu\left(B\left(x, 2^{j+1} d\right)\right)\right) \\
& \leq C \sum_{j=0}^{\infty} 2^{-j \gamma}\left(\frac{1}{\mu\left(B\left(x, 2^{j+1} d\right)\right)} \int_{B\left(x, 2^{j+1} d\right)}\left|b(y)-b_{2^{j+1} d}(x)\right|^{p^{\prime}} d \mu(y)\right. \\
& \left.+\left((j+1)\|b\|_{*, 1}\right)^{p^{\prime}}\right) \leq\|b\|_{*, p^{\prime}}^{p^{\prime}}
\end{aligned}
$$

On the other hand, if $\delta\left(x_{0}, y\right) \leq \beta d$ and $\delta(x, y) \leq d$, then $\delta\left(x_{0}, y\right) \geq((1-k \beta) / k) d$ and $\delta\left(x_{0}, y\right) \leq k(\beta+1) \delta(x, y)$. Hence, by invoking again the normality of $(X, \delta, \mu)$ we can write 


$$
\begin{aligned}
& d^{\gamma} \int_{X \backslash B(x, d)} \frac{|f(y)|^{p}}{\delta(x, y)^{1+\gamma-p \alpha}} d \mu(y) \\
& \quad \leq C d^{\gamma} \int_{X \backslash B\left(x_{0},((1-k \beta) / k) d\right)} \frac{|f(y)|^{p}}{\delta\left(x_{0}, y\right)^{1+\gamma-p \alpha}} d \mu(y) \\
& \quad \leq C d^{\gamma} \sum_{j=0}^{\infty} \int_{2^{j+1}((1-k \beta) / k) d>\delta\left(x_{0}, y\right) \geq 2^{j}((1-k \beta) / k) d} \frac{|f(y)|^{p}}{\delta\left(x_{0}, y\right)^{1+\gamma-p \alpha}} d \mu(y) \\
& \quad \leq C d^{\gamma} \sum_{j=0}^{\infty}\left(d 2^{j}\right)^{-1-\gamma+p \alpha} \int_{B\left(x_{0}, 2^{j+1}((1-k \beta) / k) d\right)}|f(y)|^{p} d \mu(y) \\
& \quad \leq C \sum_{j=0}^{\infty} 2^{-j \gamma} \frac{1}{\mu\left(B\left(x_{0}, 2^{j+1}((1-k \beta) / k) d\right)\right)^{1-p \alpha}} \int_{B\left(x_{0}, 2^{j+1}((1-k \beta) / k) d\right)}|f(y)|^{p} d \mu(y) \\
& \quad \leq C M_{p \alpha}\left(|f|^{p}\right)\left(x_{0}\right) .
\end{aligned}
$$

Thus the result is proved.

The following Whitney type covering lemma will be useful in the sequel.

LEMMA 2.5 (see [4, Lemma 1] and [13, Lemma 2]). Let $\Omega$ be a proper open subset of $X$ and let $B$ be a ball in $X$ such that $B \cap \Omega \neq \varnothing$ and $B \cap(X \backslash \Omega) \neq \varnothing$. Then there exists $a$ sequence $\left(B_{j}\right)_{j \in \mathbb{N}}$ of balls in $X$ satisfying the following three properties:

(i) $\Omega \cap B \subset \cup_{j \in \mathbb{N}} B_{j} \subset \Omega \cap\left(B^{*}\right)^{*}$,

(ii) $B_{j}^{*} \cap(X \backslash \Omega) \neq \varnothing, j \in \mathbb{N}$, and

(iii) $\mu(\Omega \cap B) \leq \sum_{j=1}^{\infty} \mu\left(B_{j}\right) \leq C \mu\left(\Omega \cap\left(B^{*}\right)^{*}\right)$.

Here if $B=B(x, r)$, with $x \in X$ and $r>0, B^{*}$ denotes the ball $B(x, r k(2 k+1))$.

Next we will prove in the main lemma a good- $\lambda$ inequality.

LEMMA 2.6. Let $0 \leq \rho<1$ and $1<p<1 / \alpha$. Assume that $(X, \delta, \mu)$ is a normal space of homogeneous type that is of order $\rho$ and such that $\mu(\{x\})=0, x \in X$. Let $b \in B M O$ and $f$ be a measurable function on $X$. Then there exists $\beta_{0}$ such that for every $\beta \geq \beta_{0}$ and $\gamma>1$

$$
\begin{aligned}
\mu(\{x \in X: C(b, f)(x) & \left.\left.>\beta \lambda,\|b\|_{*, p^{\prime}}\left(I_{\alpha}(|f|)(x)+\left(M_{p \alpha}\left(|f|^{p}\right)(x)\right)^{1 / p}\right) \leq \gamma \lambda\right\}\right) \\
& \leq C \gamma \mu(\{x \in X: C(b, f)(x)>\lambda\}),
\end{aligned}
$$

provided that one of the following two conditions holds:

(i) $\lambda>0$ and $\mu(X)=\infty$,

(ii) $\lambda>\left(C_{0} / \mu(X)\right)^{1-\alpha}\|b\|_{p^{\prime}}\|f\|_{p}$ and $\mu(X)<\infty$, where $C_{0}$ is the positive constant appearing in Lemma 2.3.

Proof. Let $\beta, \gamma>0$ and $\lambda$ satisfying the imposed conditions. We define the following sets:

$$
\begin{aligned}
E_{\lambda}(\beta, \gamma)= & \{x \in X: C(b, f)(x)>\beta \gamma, \\
& \left.\|b\|_{*, p^{\prime}}\left(I_{\alpha}(|f|)(x)+\left(M_{p \alpha}\left(|f|^{p}\right)(x)\right)^{1 / p}\right) \leq \gamma \lambda\right\}, \\
W_{\lambda}= & \{x \in X: C(b, f)(x)>\lambda\} .
\end{aligned}
$$


Note that we can assume, without loss of generality, that $W_{\lambda} \neq \varnothing$ and $W_{\lambda} \neq X$. Indeed, suppose firstly that $\mu(X)=\infty$. If $W_{\lambda}=X$, then (2.14) is clear for every $\beta>0$ and $\gamma>0$. On the other hand, if $\mu(X)<\infty$, then Lemma 2.3 implies that $\mu\left(W_{\lambda}\right)<\mu(X)$ when $\lambda>\left(C_{0} / \mu(X)\right)^{1-\alpha}\|b\|_{p^{\prime}}\|f\|_{p}$ and where $C_{0}$ is the positive constant that appears in Lemma 2.3. Also if $\mu(X) \leq \infty$ and $W_{\lambda}=\varnothing$, then (2.14) holds for every $\beta>1$ and $\gamma>0$.

Let $B$ be a ball in $X$ such that $B \cap W_{\lambda} \neq \varnothing$ and $B \cap\left(X \backslash W_{\lambda}\right) \neq \varnothing$. Then there exists a sequence $\left(B_{j}\right)_{j=1}^{\infty}$ of balls in $X$ satisfying conditions (i), (ii), and (iii) in Lemma 2.5 by replacing $\Omega$ by $W_{\lambda}$.

Let $j \in \mathbb{N}$. Write $B_{j}=B(a, d)$, with $a \in X$ and $d>0$. We define $B_{j}^{1}=B\left(a, \alpha_{1} d\right)$ and $B_{j}^{2}=B\left(a, \alpha_{2} d\right)$, where $\alpha_{1} \leq k\left(2 k^{2}(1+k(2 k+1))+1\right)$ and $\alpha_{2}>k\left(1+k\left(\alpha_{1}+1\right)\right)$.

Assume that $B_{j} \cap E_{\lambda}(\beta, \gamma) \neq \varnothing$ and choose $x_{1} \in B_{j} \cap E_{\lambda}(\beta, \gamma)$. We write $f=f_{1}+$ $f_{2}$, where $f_{1}=f X_{B_{j}^{1}}$, and $b=b_{1}+b_{2}$, being $b_{1}=\left(b-b_{B_{j}^{2}}\right) X_{B_{j}^{2}}$ and $b_{B_{j}^{2}}=1 / \mu\left(B_{j}^{2}\right)$ $\times \int_{B_{j}^{2}} b(y) d \mu(y)$.

We have that $C\left(b, f_{1}\right)(x) \leq C\left(b_{1}, f_{1}\right)(x)$, for every $x \in B_{j}$. Indeed, let $x \in B_{j}$ and $\epsilon>0$. Since $\alpha_{2}>k\left(1+k\left(\alpha_{1}+1\right)\right)$, if $B(x, \epsilon) \cap\left(X \backslash B_{j}^{2}\right) \neq \varnothing$, then $B_{j}^{1} \subset B(x, \epsilon)$. Hence we can write

$$
\begin{aligned}
\left(b_{1}\right)_{\epsilon}(x) & =\frac{1}{\mu(B(x, \epsilon))} \int_{B(x, \epsilon)} b_{1}(y) d \mu(y) \\
& =\frac{1}{\mu(B(x, \epsilon))} \int_{B(x, \epsilon) \cap B_{j}^{2}}\left(b(y)-b_{B_{j}^{2}}\right) d \mu(y) \\
& =\frac{1}{\mu(B(x, \epsilon))} \int_{B(x, \epsilon)} b(y) d \mu(y)-b_{B_{j}^{2}}=b_{\epsilon}(x)-b_{B_{j}^{2}},
\end{aligned}
$$

provided that $B_{j}^{1} \cap(X \backslash B(x, \epsilon)) \neq \varnothing$.

Then, since $B_{j}^{1} \subset B_{j}^{2}$, one has

$$
\begin{aligned}
C\left(b, f_{1}\right)(x) & =\sup _{\epsilon>0}\left|\int_{X \backslash B(x, \epsilon)} \frac{b(y)-b_{\epsilon}(x)}{\delta(x, y)^{1-\alpha}} f_{1}(y) d \mu(y)\right| \\
& =\sup _{\epsilon>0}\left|\int_{(X \backslash B(x, \epsilon)) \cap B_{j}^{1}} \frac{b_{1}(y)+b_{B_{j}^{2}}-b_{\epsilon}(x)}{\delta(x, y)^{1-\alpha}} f_{1}(y) d \mu(y)\right| \\
& \leq \sup _{\epsilon>0}\left|\int_{X \backslash B(x, \epsilon)} \frac{b_{1}(y)-\left(b_{1}\right)_{\epsilon}(x)}{\delta(x, y)^{1-\alpha}} f_{1}(y) d \mu(y)\right|=C\left(b_{1}, f_{1}\right)(x) .
\end{aligned}
$$

Moreover from Lemma 2.3 we deduce that for every $\beta>1$,

$$
\begin{aligned}
& \mu\left(\left\{x \in B_{j}: C\left(b_{1}, f_{1}\right)(x)>\beta \lambda\right\}\right) \\
& \leq C\left(\frac{\left\|b_{1}\right\|_{p^{\prime}}\left\|f_{1}\right\|_{p}}{\beta \lambda}\right)^{1 / 1-\alpha} \\
& =C\left(\int_{B_{j}^{2}}\left|b(y)-b_{B_{j}^{2}}\right|^{p^{\prime}} d \mu(y)\right)^{1 / p^{\prime}(1-\alpha)}\left(\int_{B_{j}^{1}}|f(y)|^{p} d \mu(y)\right)^{1 / p(1-\alpha)} \\
& \leq C \lambda^{1 / \alpha-1} \mu\left(B_{j}\right)\left(\|b\|_{*, p^{\prime}}\left(M_{p \alpha}\left(|f|^{p}\right)\left(x_{1}\right)\right)^{1 / p}\right)^{1 / 1-\alpha},
\end{aligned}
$$

because $\mu$ is doubling. 
Hence, since $x_{1} \in B_{j} \cap E_{\lambda}(\beta, \gamma)$ if $\gamma<1$, then

$$
\mu\left(\left\{x \in B_{j}: C\left(b, f_{1}\right)(x)>\beta \lambda\right\}\right) \leq C \gamma \mu\left(B_{j}\right) .
$$

By virtue of (ii) in Lemma $2.5, B_{j}^{*} \cap\left(X \backslash W_{\lambda}\right) \neq \varnothing$. Choose $x_{0} \in B_{j}^{*} \cap\left(X \backslash W_{\lambda}\right)$, that is, $x_{0} \in B_{j}^{*}$ and $C(b, f)\left(x_{0}\right) \leq \lambda$.

Now our objective is to estimate

$$
\mu\left(\left\{x \in B_{j}: C\left(b, f_{2}\right)(x)>\beta \lambda\right\}\right) .
$$

We consider two cases.

Assume firstly that $\epsilon>\sigma d$, where $\alpha_{2} / k-1>\sigma>\left(\alpha_{1}+1\right) k$. Since $\sigma>\left(\alpha_{1}+1\right) k$, for every $x \in B_{j}, B_{j}^{1} \subset B(x, \epsilon)$. Let $x \in B_{j}$. We have

$$
\begin{aligned}
\int_{X \backslash B(x, \epsilon)} \frac{b(y)-b_{\epsilon}(x)}{\delta(x, y)^{1-\alpha}} f_{2}(y) d \mu(y) & =\int_{X \backslash B(x, \epsilon)} \frac{b(y)-b_{\epsilon}(x)}{\delta(x, y)^{1-\alpha}} f(y) d \mu(y) \\
& =I_{1}+I_{2}+I_{3},
\end{aligned}
$$

where

$$
\begin{aligned}
I_{1} & =\int_{X \backslash B(x, \epsilon)} \frac{b(y)-b_{\epsilon}(x)}{\delta(x, y)^{1-\alpha}} f(y) d \mu(y)-\int_{X \backslash B(x, \epsilon)} \frac{b(y)-b_{\epsilon}\left(x_{0}\right)}{\delta(x, y)^{1-\alpha}} f(y) d \mu(y), \\
I_{2} & =\int_{X \backslash B(x, \epsilon)} \frac{b(y)-b_{\epsilon}\left(x_{0}\right)}{\delta(x, y)^{1-\alpha}} f(y) d \mu(y)-\int_{X \backslash B\left(x_{0}, \epsilon\right)} \frac{b(y)-b_{\epsilon}\left(x_{0}\right)}{\delta\left(x_{0}, y\right)^{1-\alpha}} f(y) d \mu(y), \\
I_{3} & =\int_{X \backslash B\left(x_{0}, \epsilon\right)} \frac{b(y)-b_{\epsilon}\left(x_{0}\right)}{\delta\left(x_{0}, y\right)^{1-\alpha}} f_{2}(y) d \mu(y) .
\end{aligned}
$$

We are going to estimate $I_{i}, i=1,2,3$.

As mentioned above if $\delta(x, y)>\epsilon$, then $y \notin B_{j}^{1}$. Hence $\delta(x, y)>\epsilon$ implies that $\delta(x, y) \geq\left(\left(\alpha_{1}-k\right) / k\right) d>0$. Then we can write

$$
\frac{\delta\left(x_{1}, y\right)}{\delta(x, y)} \leq \frac{k\left(\delta\left(x, x_{1}\right)+\delta(x, y)\right)}{\delta(x, y)} \leq \frac{2 k^{3}}{\alpha_{1}-k}+k
$$

provided that $\delta(x, y)>\epsilon$.

Therefore it follows

$$
\begin{aligned}
\left|I_{1}\right| & =\left|\int_{X \backslash B(x, \epsilon)} \frac{b(y)-b_{\epsilon}(x)}{\delta(x, y)^{1-\alpha}} f(y) d \mu(y)-\int_{X \backslash B(x, \epsilon)} \frac{b(y)-b_{\epsilon}\left(x_{0}\right)}{\delta(x, y)^{1-\alpha}} f(y) d \mu(y)\right| \\
& \leq \int_{X \backslash B(x, \epsilon)} \frac{\left|b_{\epsilon}\left(x_{0}\right)-b_{\epsilon}(x)\right|}{\delta(x, y)^{1-\alpha}}|f(y)| d \mu(y) \\
& \leq C\left|b_{\epsilon}\left(x_{0}\right)-b_{\epsilon}(x)\right| \int_{X \backslash B_{j}^{1}} \frac{|f(y)|}{\delta\left(x_{1}, y\right)^{1-\alpha}} d \mu(y) \\
& \leq C\left|b_{\epsilon}\left(x_{0}\right)-b_{\epsilon}(x)\right| I_{\alpha}(|f|)\left(x_{1}\right) .
\end{aligned}
$$

Moreover if $y \in B\left(x_{0}, \epsilon\right)$, then

$$
\delta(x, y) \leq k\left(\delta\left(y, x_{0}\right)+\delta\left(x_{0}, x\right)\right) \leq k(\epsilon+k d(1+k(2 k+1)))<2^{m} \epsilon,
$$


where $m \in \mathbb{N}$ is large enough and $m$ is not depending on $d$ and $\epsilon$.

Hence, since $(X, \delta, \mu)$ is normal we have that

$$
\begin{aligned}
& \left|b_{\epsilon}\left(x_{0}\right)-b_{\epsilon}(x)\right| \\
& \quad \leq \frac{1}{\mu\left(B\left(x_{0}, \epsilon\right)\right)} \int_{B\left(x_{0}, \epsilon\right)}\left|b(y)-b_{\epsilon}(x)\right| d \mu(y) \\
& \quad \leq C \frac{1}{\mu\left(B\left(x, 2^{m} \epsilon\right)\right)} \int_{B\left(x, 2^{m} \epsilon\right)}\left|b(y)-b_{\epsilon}(x)\right| d \mu(y) \\
& \quad \leq C\left(\frac{1}{\mu\left(B\left(x, 2^{m} \epsilon\right)\right)} \int_{B\left(x, 2^{\left.m_{\epsilon}\right)}\right.}\left|b(y)-b_{2^{m}}(x)\right| d \mu(y)+\left|b_{2^{m}}(x)-b_{\epsilon}(x)\right|\right) \\
& \quad \leq C\|b\|_{*, p^{\prime} .}
\end{aligned}
$$

Thus we conclude that

$$
\left|I_{1}\right| \leq C\|b\|_{*, p^{\prime}} I_{\alpha}(|f|)\left(x_{1}\right) \leq C \gamma \lambda \text {. }
$$

On the other hand, to estimate $I_{2}$ we will use that $(X, \delta, \mu)$ is a space of homogeneous type which is of order $\rho \in(0,1)$. It is clear that

$$
\begin{aligned}
\left|I_{2}\right| \leq & \int_{\delta(x, y) \geq \epsilon \text { and } \delta\left(x_{0}, y\right) \geq \epsilon}\left|b(y)-b_{\epsilon}\left(x_{0}\right)\right|\left|f_{2}(y)\right|\left|\delta(x, y)^{\alpha-1}-\delta\left(x_{0}, y\right)^{\alpha-1}\right| d \mu(y) \\
& +\mid \int_{\delta(x, y) \geq \epsilon \text { and } \delta\left(x_{0}, y\right)<\epsilon} \frac{b(y)-b_{\epsilon}\left(x_{0}\right)}{\delta(x, y)^{1-\alpha}} f_{2}(y) d \mu(y) \\
& \quad-\int_{\delta\left(x_{0}, y\right) \geq \epsilon \text { and } \delta(x, y)<\epsilon} \frac{b(y)-b_{\epsilon}\left(x_{0}\right)}{\delta\left(x_{0}, y\right)^{1-\alpha}} f_{2}(y) d \mu(y) \mid .
\end{aligned}
$$

Note that, since $\sigma>2 k^{2}(1+k(2 k+1)), \delta\left(x_{0}, y\right) \leq 2 k \delta\left(x_{0}, x\right)$ provided that $\delta\left(x_{0}, y\right)>$ $\epsilon$. Hence, according to [11, Lemma II.3] and Lemma 2.4, since $\delta\left(x_{0}, x_{1}\right)<(1 / 2 k) \epsilon$, we have,

$$
\begin{aligned}
& \int_{\delta(x, y) \geq \epsilon} \text { and } \delta\left(x_{0}, y\right) \geq \epsilon \\
& \leq C \delta\left(x, x_{0}\right)^{\rho} \int_{X \backslash B\left(x_{0}, \epsilon\right)}\left|b(y)-b_{\epsilon}\left(x_{0}\right)\right|\left|f_{2}(y)\right|\left|\delta(x, y)^{\alpha-1}-\delta\left(x_{0}, y\right)^{\alpha-1}\right| d \mu(y) \\
& \leq C \epsilon^{\rho} \int_{X \backslash B\left(x_{0}, \epsilon\right)}\left|b(y)-b_{\epsilon}\left(x_{0}\right)\right|\left|f_{2}(y)\right|\left|\delta\left(x_{0}, y\right)^{\alpha-\rho-1}\right| d \mu(y)|| \delta\left(x_{0}, y\right)^{\alpha-\rho-1} \mid d \mu(y) \\
& \leq C\|b\|_{*, p^{\prime}}\left(M_{p \alpha}\left(|f|^{p}\right)\left(x_{1}\right)\right)^{1 / p} \leq C \gamma \lambda .
\end{aligned}
$$

Moreover, $\delta(x, y)<\epsilon$ implies that $\delta\left(x_{0}, y\right) \leq \epsilon(k+(1 / 2))$ and this inequality implies that $\delta\left(x_{1}, y\right) \leq \epsilon(k(k+(1 / 2))+(1 / 2))$. Then, by taking into account the normality of 
$(X, \delta, \mu)$, Holder inequality leads to

$$
\begin{aligned}
& \mid \int_{\delta(x, y) \geq \epsilon \text { and } \delta\left(x_{0}, y\right)<\epsilon} \frac{b(y)-b_{\epsilon}\left(x_{0}\right)}{\delta(x, y)^{1-\alpha}} f_{2}(y) d \mu(y) \\
& \quad-\int_{\delta\left(x_{0}, y\right) \geq \epsilon \text { and } \delta(x, y)<\epsilon} \frac{b(y)-b_{\epsilon}\left(x_{0}\right)}{\delta\left(x_{0}, y\right)^{1-\alpha}} f_{2}(y) d \mu(y) \mid \\
& \leq C \epsilon^{\alpha-1} \int_{B\left(x_{0}, \epsilon(k+(1 / 2))\right)}\left|b(y)-b_{\epsilon}\left(x_{0}\right)\right|\left|f_{2}(y)\right| d \mu(y) \\
& \leq C \frac{1}{\mu\left(B\left(x_{0}, \epsilon(k+(1 / 2))\right)\right)^{1-\alpha}} \int_{B\left(x_{0}, \epsilon(k+(1 / 2))\right)}\left|b(y)-b_{\epsilon}\left(x_{0}\right)\right|\left|f_{2}(y)\right| d \mu(y) \\
& \leq\|b\|_{*, p^{\prime}}\left(M_{p \alpha}\left(|f|^{p}\right)\left(x_{1}\right)\right)^{1 / p} \leq C \gamma \lambda .
\end{aligned}
$$

Finally, since $x_{0} \notin W_{\lambda}$, we have

$$
\left|I_{3}\right| \leq \lambda
$$

By combining (2.21), (2.27), and (2.31) we conclude that

$$
\sup _{\epsilon>d \sigma}\left|\int_{X \backslash B(x, \epsilon)} \frac{b(y)-b_{\epsilon}(x)}{\delta(x, y)^{1-\alpha}} f_{2}(y) d \mu(y)\right| \leq C \gamma \lambda+\lambda .
$$

Assume now $0<\epsilon \leq d \sigma$. Let $x \in B_{j}$. It is not hard to see that if $y$ is in the support of $f_{2}$ then $\delta(x, y) \geq\left(\left(\alpha_{1}-k\right) / k\right) d$ and $\delta\left(x_{0}, y\right) \geq\left(\left(\alpha_{1}-k^{2}(2 k+1)\right) / k\right) d$. We choose $\omega>0$ such that $\omega<\left(\alpha_{1}-k\right) / k$.

We can write

$$
\int_{X \backslash B(x, \epsilon)} \frac{b(y)-b_{\epsilon}(x)}{\delta(x, y)^{1-\alpha}} f_{2}(y) d \mu(y)=J_{1}+J_{2}+J_{3},
$$

where

$$
\begin{aligned}
J_{1} & =\int_{X \backslash B(x, \epsilon)} \frac{b_{\omega d}\left(x_{0}\right)-b_{\epsilon}(x)}{\delta(x, y)^{1-\alpha}} f_{2}(y) d \mu(y), \\
J_{2} & =\int_{X \backslash B(x, \epsilon)} \frac{b(y)-b_{\omega d}\left(x_{0}\right)}{\delta(x, y)^{1-\alpha}} f_{2}(y) d \mu(y)-\int_{X \backslash B\left(x_{0}, \epsilon\right)} \frac{b(y)-b_{\omega d}\left(x_{0}\right)}{\delta\left(x_{0}, y\right)^{1-\alpha}} f_{2}(y) d \mu(y), \\
J_{3} & =\int_{X \backslash B\left(x_{0}, \epsilon\right)} \frac{b(y)-b_{\omega d}\left(x_{0}\right)}{\delta\left(x_{0}, y\right)^{1-\alpha}} f_{2}(y) d \mu(y) .
\end{aligned}
$$

We will estimate $J_{i}, i=1,2,3$.

By proceeding as in the study of $I_{1}$, since $k(\sigma+1)<\alpha_{2}$, we obtain

$$
\begin{aligned}
\left|J_{1}\right| & \leq C\left|b_{\omega d}\left(x_{0}\right)-b_{\epsilon}(x)\right| I_{\alpha}(|f|)\left(x_{1}\right) \\
& \leq C \frac{1}{\mu(B(x, \epsilon))} \int_{B(x, \epsilon)}\left|b(y)-b_{\omega d}\left(x_{0}\right)\right| x_{B_{j}^{2}}(y) d \mu(y) I_{\alpha}(|f|)\left(x_{1}\right) \\
& \leq C M\left(\left(b-b_{\omega d}\left(x_{0}\right)\right) x_{B_{j}^{2}}\right)(x) I_{\alpha}(|f|)\left(x_{1}\right) .
\end{aligned}
$$


On the other hand, we have that

$$
\begin{aligned}
J_{2}= & \int_{X \backslash B(x, \epsilon)} \frac{b(y)-b_{\omega d}\left(x_{0}\right)}{\delta(x, y)^{1-\alpha}} f_{2}(y) d \mu(y)-\int_{X \backslash B\left(x_{0}, \epsilon\right)} \frac{b(y)-b_{\omega d}\left(x_{0}\right)}{\delta(x, y)^{1-\alpha}} f_{2}(y) d \mu(y) \\
= & \int_{\delta(x, y) \geq \epsilon \text { and } \delta\left(x_{0}, y\right) \geq \epsilon}\left(b(y)-b_{\omega d}\left(x_{0}\right)\right) f_{2}(y)\left(\delta(x, y)^{\alpha-1}-\delta\left(x_{0}, y\right)^{\alpha-1}\right) d \mu(y) \\
& +\int_{\delta(x, y) \geq \epsilon \text { and } \delta\left(x_{0}, y\right)<\epsilon} \frac{b(y)-b_{\omega d}\left(x_{0}\right)}{\delta(x, y)^{1-\alpha}} f_{2}(y) d \mu(y) \\
& -\int_{\delta(x, y)<\epsilon \text { and } \delta\left(x_{0}, y\right) \geq \epsilon} \frac{b(y)-b_{\omega d}\left(x_{0}\right)}{\delta\left(x_{0}, y\right)^{1-\alpha}} f_{2}(y) d \mu(y) .
\end{aligned}
$$

Since $(X, \delta, \mu)$ is a space of homogeneous type of order $\rho \in(0,1)$, by virtue of [11, Lemma 2.3], we have

$$
\begin{aligned}
& \left|\int_{\delta(x, y) \geq \epsilon \text { and } \delta\left(x_{0}, y\right) \geq \epsilon}\left(b(y)-b_{\omega d}\left(x_{0}\right)\right) f_{2}(y)\left(\delta(x, y)^{\alpha-1}-\delta\left(x_{0}, y\right)^{\alpha-1}\right) d \mu(y)\right| \\
& \quad \leq C \delta\left(x, x_{0}\right)^{\rho} \int_{\delta(x, y) \geq \epsilon \text { and } \delta\left(x_{0}, y\right) \geq \epsilon}\left|b(y)-b_{\omega d}\left(x_{0}\right)\right|\left|f_{2}(y)\right| \delta(x, y)^{\alpha-\rho-1} d \mu(y),
\end{aligned}
$$

because if $y$ is in the support of $f_{2}$, then $\delta(x, y) \geq\left(\left(\alpha_{1}-k\right) / k\right) d \geq 2 k^{2}(k(2 k+1)+$ $1) d \geq 2 k \delta\left(x_{0}, x\right)$. Hence, since $y \in \operatorname{supp} f_{2}$ implies that $\delta\left(x_{1}, y\right)>\omega d$, by proceeding as in the proof of Lemma 2.4 we conclude

$$
\begin{aligned}
& \mid \int_{\delta(x, y) \geq \epsilon \text { and } \delta\left(x_{0}, y\right) \geq \epsilon}\left(b(y)-b_{\omega d}\left(x_{0}\right)\right) f_{2}(y)\left(\delta(x, y)^{\alpha-1}-\delta\left(x_{0}, y\right)^{\alpha-1}\right) d \mu(y) \mid \\
& \leq C \delta\left(x, x_{0}\right)^{\rho} \int_{\delta\left(x_{1}, y\right)>\omega d} \frac{\left|b(y)-b_{\omega d}\left(x_{0}\right)\right|}{\delta\left(x_{1}, y\right)^{1+\rho-\alpha}}|f(y)| d \mu(y) \\
& \leq C\|b\|_{*, p^{\prime}}\left(M_{p \alpha}\left(|f|^{p}\right)\left(x_{1}\right)\right)^{1 / p} \leq C \gamma \lambda .
\end{aligned}
$$

Also, since if $\delta(x, y)<\epsilon$, then $\delta\left(x_{0}, y\right)<d k(k(k(2 k+1)+1)+\sigma)$ and since $\omega<\alpha_{1}$, we have

$$
\begin{aligned}
& \mid \int_{\delta(x, y) \geq \epsilon \text { and } \delta\left(x_{0}, y\right)<\epsilon} \frac{b(y)-b_{\omega d}\left(x_{0}\right)}{\delta(x, y)^{1-\alpha}} f_{2}(y) d \mu(y) \\
& \quad-\int_{\delta(x, y)<\epsilon \text { and } \delta\left(x_{0}, y\right) \geq \epsilon} \frac{b(y)-b_{\omega d}\left(x_{0}\right)}{\delta\left(x_{0}, y\right)^{1-\alpha}} f_{2}(y) d \mu(y) \mid \\
& \leq \int_{\omega d<\delta\left(x_{0}, y\right)<k(k(k(2 k+1)+1)+\sigma) d}\left|b(y)-b_{\omega d}\left(x_{0}\right)\right|\left|f_{2}(y)\right| \\
& \quad \times\left(\delta(x, y)^{\alpha-1}+\delta\left(x_{0}, y\right)^{\alpha-1}\right) d \mu(y) \\
& \leq C \int_{\omega d<\delta\left(x_{0}, y\right)<k(k(k(2 k+1)+1)+\sigma) d} \frac{\left|b(y)-b_{\omega d}\left(x_{0}\right)\right|}{\delta\left(x_{0}, y\right)^{1-\alpha}}\left|f_{2}(y)\right| d \mu(y) .
\end{aligned}
$$


Now by proceeding as in the proof of Lemma 2.4 we obtain that

$$
\begin{aligned}
& \mid \int_{\delta(x, y) \geq \epsilon \text { and } \delta\left(x_{0}, y\right)<\epsilon} \frac{b(y)-b_{\omega d}\left(x_{0}\right)}{\delta(x, y)^{1-\alpha}} f_{2}(y) d \mu(y) \\
& \quad-\int_{\delta(x, y)<\epsilon \text { and } \delta\left(x_{0}, y\right) \geq \epsilon} \frac{b(y)-b_{\omega d}\left(x_{0}\right)}{\delta\left(x_{0}, y\right)^{1-\alpha}} f_{2}(y) d \mu(y) \mid \\
& \leq C\|b\|_{*, p^{\prime}}\left(M_{p \alpha}\left(|f|^{p}\right)\left(x_{1}\right)\right)^{1 / p} \leq C \gamma \lambda .
\end{aligned}
$$

In a similar way we can see that

$$
\begin{aligned}
\left|J_{3}\right| & \leq \int_{X \backslash B\left(x_{0}, \omega d\right)} \frac{\left|b(y)-b_{\omega d}\left(x_{0}\right)\right|}{\delta\left(x_{0}, y\right)^{1-\alpha}}\left|f_{2}(y)\right| d \mu(y) \\
& \leq C\|b\|_{*, p^{\prime}}\left(M_{p \alpha}\left(|f|^{p}\right)\left(x_{1}\right)\right)^{1 / p} \leq C \gamma \lambda .
\end{aligned}
$$

By combining the above estimates we can conclude

$$
\begin{aligned}
\sup _{0<\epsilon \leq d \sigma} \mid \int_{X \backslash B(x, \epsilon)} & \frac{b(y)-b_{\epsilon}(x)}{\delta(x, y)^{1-\alpha}} f_{2}(y) d \mu(y) \mid \\
& \leq C\left(\lambda \gamma+I_{\alpha}(|f|)\left(x_{1}\right) M\left(\left(b-b_{\omega d}\left(x_{0}\right)\right) X_{B_{j}^{2}}\right)(x)\right) .
\end{aligned}
$$

From (2.32) and (2.42) follows that for every $x \in B_{j}$

$$
C\left(b, f_{2}\right)(x) \leq C\left(\lambda \gamma+\lambda+I_{\alpha}(|f|)\left(x_{1}\right) M\left(\left(b-b_{\omega d}\left(x_{0}\right)\right) x_{B_{j}^{2}}\right)(x)\right) .
$$

Hence if $\beta$ is large enough, then according to Lemma 2.2 and since $\mu$ is doubling

$$
\begin{aligned}
\mu(\{x \in & \left.\left.B_{j}: C\left(b, f_{2}\right)(x)>\beta \lambda\right\}\right) \\
& \leq \mu\left(\left\{x \in B_{j}: I_{\alpha}(|f|)\left(x_{1}\right) M\left(\left(b-b_{\omega d}\left(x_{0}\right)\right) x_{B_{j}^{2}}\right)(x)>\lambda\right\}\right) \\
& \leq C \lambda^{-1} I_{\alpha}(|f|)\left(x_{1}\right) \int_{B_{j}^{2}}\left|b(y)-b_{\omega d}\left(x_{0}\right)\right| d \mu(y) \\
& \leq C \lambda^{-1} I_{\alpha}(|f|)\left(x_{1}\right)\|b\|_{*, p^{\prime}} \mu\left(B_{j}\right) \leq C \gamma \mu\left(B_{j}\right) .
\end{aligned}
$$

Thus we obtain that for $\beta \geq \beta_{0}$ and $\gamma<1$, where $\beta_{0}$ is large enough,

$$
\mu\left(B_{j} \cap E_{\lambda}(\beta, \gamma)\right) \leq C \gamma \mu\left(B_{j}\right) .
$$

Hence

$$
\mu\left(B \cap E_{\lambda}(\beta, \gamma)\right) \leq C \gamma \sum_{j=1}^{\infty} \mu\left(B_{j}\right) \leq C \gamma \mu\left(W_{\lambda}\right), \quad \beta \geq \beta_{0}, \gamma<1 .
$$

Arbitrariness of $B$ allows to conclude that

$$
\mu\left(E_{\lambda}(\beta, \gamma)\right) \leq C \gamma \mu\left(W_{\lambda}\right), \quad \beta \geq \beta_{0}, \gamma<1,
$$

and the proof is finished. 
Proof OF THEOREM 1.1. To prove Theorem 1.1 we proceed as in the proof of [6, Theorem III]. We start proving that the operator $C(b, f)$ is bounded from $L^{p}(X, \mu)$ into $L^{q}(X, \mu)$, when $1<p<1 / \alpha$ and $1 / q=1 / p-\alpha$. Assume that $b \in L^{\infty}(X, \mu)$.

Let $1<p_{1}<p<1 / \alpha$ and $1 / q=1 / p-\alpha$. Assume firstly that $\mu(X)=\infty$. According to Lemma 2.6, $f \in L^{p}(X, \mu)$ we have

$$
\begin{aligned}
& \int_{X}(C(b, f)(x))^{q} d \mu(x) \\
& =\beta^{q} q \int_{0}^{\infty} \lambda^{q-1} \mu(\{x \in X: C(b, f)(x)>\beta \lambda\}) d \lambda \\
& \leq C \beta^{q}\left(\gamma \int_{0}^{\infty} \lambda^{q-1} \mu(\{x \in X: C(b, f)(x)>\lambda\}) d \lambda\right. \\
& \left.\quad+\int_{0}^{\infty} \lambda^{q-1} \mu\left(\left\{x \in X:\|b\|_{*, p^{\prime}}\left(I_{\alpha}(|f|)(x)+\left(M_{p_{1} \alpha}\left(|f|^{p_{1}}\right)(x)\right)^{1 / p_{1}}\right)>\gamma \lambda\right\}\right) d \lambda\right) \\
& =C \beta^{q}\left(\gamma \int_{X}(C(b, f)(x))^{q} d \mu(x)\right. \\
& \left.\quad+\gamma^{-q}\|b\|_{*, p^{\prime}}^{q} \int_{X}\left(I_{\alpha}(|f|)(x)+\left(M_{p_{1} \alpha}\left(|f|^{p_{1}}\right)(x)\right)^{1 / p_{1}}\right)^{q} d \mu(x)\right),
\end{aligned}
$$

provided that $\beta \geq \beta_{0}$ and $0<\gamma<1$, where $\beta_{0}$ is given in Lemma 2.6.

Hence by (2.4) and Lemma 2.1 and by taking $\gamma$ so small we can conclude that

$$
\|C(b, f)\|_{q} \leq C\|b\|_{*, p^{\prime}}\left(\left\|I_{\alpha}(|f|)\right\|_{q}+\left\|M_{p_{1} \alpha}\left(|f|^{p_{1}}\right)\right\|_{q / p_{1}}^{1 / p_{1}}\right) .
$$

According to Lemmas 2.1 and 2.2 it follows

$$
\|C(b, f)\|_{q} \leq C\|b\|_{*, p^{\prime}}\|f\|_{p} .
$$

Suppose now that $\mu(X)<\infty$. Since $C(b, f)=C(b-a, f)$, for every $a \in \mathbb{C}$, we can assume, without loss of generality, that $\int_{X} b d \mu=0$. Then Lemma 2.6 leads, for every $f \in L^{p}(X, \mu)$, to

$$
\begin{aligned}
& \int_{X}(C(b, f)(x))^{q} d \mu(x) \\
& =\beta^{q} q \int_{0}^{\infty} \lambda^{q-1} \mu(\{x \in X: C(b, f)(x)>\beta \lambda\}) d \lambda \\
& \leq C \beta^{q}\left(\int_{0}^{\|b\|_{p^{\prime}}\|f\|_{p}\left(C_{0} / \mu(X)\right)^{1-\alpha}} \lambda^{q-1} d \lambda+\gamma \int_{X}(C(b, f)(x))^{q} d \mu(x)\right. \\
& \left.\quad+\gamma^{-q}\|b\|_{*, p^{\prime}}^{q} \int_{X}\left(I_{\alpha}(|f|)(x)+\left(M_{p_{1} \alpha}\left(|f|^{p_{1}}\right)(x)\right)^{1 / p_{1}}\right)^{q} d \mu(x)\right) \\
& \leq C \beta^{q}\left(\|b\|_{p^{\prime}}^{q}\|f\|_{p}^{q}+\gamma \int_{X}(C(b, f)(x))^{q} d \mu(x)\right. \\
& \left.\quad+\gamma^{-q}\|b\|_{*, p^{\prime}}^{q} \int_{X}\left(I_{\alpha}(|f|)(x)+\left(M_{p_{1} \alpha}\left(|f|^{p_{1}}\right)(x)\right)^{1 / p_{1}}\right)^{q} d \mu(x)\right),
\end{aligned}
$$

when $\beta \geq \beta_{0}$ and $0<\gamma<1, \beta_{0}$ being as in Lemma 2.6.

Thus we deduce from Lemmas 2.1 and 2.2 that

$$
\|C(b, f)\|_{q} \leq C\|b\|_{*, p^{\prime}}\|f\|_{p}
$$


Now we note that

$$
\begin{aligned}
{\left[b, I_{\alpha}\right](f)(x) } & =\lim _{\epsilon \rightarrow 0^{+}}\left(b(x) \int_{X \backslash B(x, \epsilon)} \frac{f(y)}{\delta(x, y)^{1-\alpha}} d \mu(y)-\int_{X \backslash B(x, \epsilon)} \frac{b(y) f(y)}{\delta(x, y)^{1-\alpha}} d \mu(y)\right) \\
& =\lim _{\epsilon \rightarrow 0^{+}}\left(\left(b(x)-b_{\epsilon}(x)\right) \int_{X \backslash B(x, \epsilon)} \frac{f(y)}{\delta(x, y)^{1-\alpha}} d \mu(y)\right. \\
& \left.-\int_{X \backslash B(x, \epsilon)} \frac{b(y)-b_{\epsilon}(x)}{\delta(x, y)^{1-\alpha}} f(y) d \mu(y)\right) \\
= & -\lim _{\epsilon \rightarrow 0^{+}} \int_{X \backslash B(x, \epsilon)} \frac{b(y)-b_{\epsilon}(x)}{\delta(x, y)^{1-\alpha}} f(y) d \mu(y),
\end{aligned}
$$

for every $f \in L^{p}(X, \mu)$, and a.e. $x \in X$.

Then

$$
\left\|\left[b, I_{\alpha}\right]\right\|_{q} \leq\|C(b, f)\|_{q}
$$

for each $f \in L^{p}(X, \mu)$.

To finish the proof it is sufficient to take into account [3, Lemma 2.5] and Fatou's lemma.

ACKNOWLEDGEMENT. The research was partially supported by DGICYT Grant PB 94-0591 (Spain).

\section{REFERENCES}

[1] A. Bernardis and O. Salinas, Two-weight norm inequalities for the fractional maximal operator on spaces of homogeneous type, Studia Math. 108 (1994), no. 3, 201-207. MR 94m:42038. Zbl 846.42013.

[2] M. Bramanti, Commutators of integral operators with positive kernels, Matematiche (Catania) 49 (1994), no. 1, 149-168 (1995). MR 97c:47059. Zbl 840.42009.

[3] M. Bramanti and M. C. Cerutti, Commutators of singular integrals and fractional integrals on homogeneous spaces, Harmonic Analysis and Operator Theory (Caracas, 1994) (Providence, RI), Contemp. Math., 189, Amer. Math. Soc., 1995, pp. 81-94. MR 96m:42024. Zbl 837.43013.

[4] N. Burger, Espace des fonctions a variation moyenne bornee sur un espace de nature homogene, C. R. Acad. Sci. Paris Sér. A-B 286 (1978), no. 3, A139-A142. MR 57\#7041. Zbl 368.46037.

[5] S. Chanillo, A note on commutators, Indiana Univ. Math. J. 31 (1982), no. 1, 7-16. MR 84j:42027. Zbl 523.42015.

[6] R. R. Coifman and C. Fefferman, Weighted norm inequalities for maximal functions and singular integrals, Studia Math. 51 (1974), 241-250. MR 50\#10670. Zbl 291.44007.

[7] R. R. Coifman, R. Rochberg, and G. Weiss, Factorization theorems for Hardy spaces in several variables, Ann. of Math. (2) 103 (1976), no. 3, 611-635. MR 54\#843. Zbl 326.32011.

[8] R. R. Coifman and G. Weiss, Analyse Harmonique Non-Commutative sur Certains Espaces Homogenes, Lecture Notes in Mathematics, Vol. 242, Springer-Verlag, Berlin, New York, 1971. MR 58\#17690. Zbl 224.43006.

[9] _ Extensions of Hardy spaces and their use in analysis, Bull. Amer. Math. Soc. 83 (1977), no. 4, 569-645. MR 56\#6264. Zbl 358.30023.

[10] A. E. Gatto, C. Segovia, and S. Vagi, On fractional differentiation and integration on spaces of homogeneous type, Rev. Mat. Iberoamericana 12 (1996), no. 1, 111-145. MR 97k:42028. Zbl 921.43005. 
[11] A. E. Gatto and S. Vagi, Fractional integrals on spaces of homogeneous type, Analysis and Partial Differential Equations (New York), Dekker, 1990, pp. 171-216. MR 91e:42032. Zbl 695.43006.

[12] V. M. Kokilashvili and A. Kufner, Fractional integrals on spaces of homogeneous type, Comment. Math. Univ. Carolin. 30 (1989), no. 3, 511-523. MR 91e:42027. Zbl 686.42013.

[13] R. L. Long and L. Yang, BMO functions in spaces of homogeneous type, Sci. Sinica Ser. A 27 (1984), no. 7, 695-708. MR 85m:42022. Zbl 572.42011.

[14] J. Stromberg and A. Torchinsky, Weighted Hardy Spaces, Lecture Notes in Mathematics, 1381, Springer-Verlag, Berlin, 1989. MR 90j:42053. Zbl 676.42021.

[15] R. L. Wheeden, A characterization of some weighted norm inequalities for the fractional maximal function, Studia Math. 107 (1993), no. 3, 257-272. MR 94m:42044. Zbl 809.42009.

JoRge J. BETANCOR: DEPARTAMENTO DE ANÁLISIS MATEMÁTICO, UNIVERSIDAD DE LA LAGUNA, 38271-LA LAGUNA, TENERIFE ISLAS CANARIAS, ESPAÑA

E-mail address: jbetanco@u11.es 


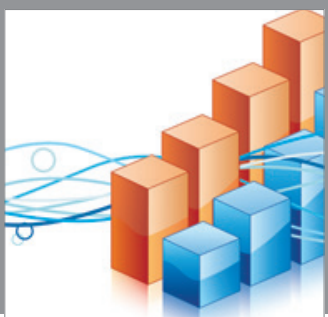

Advances in

Operations Research

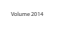

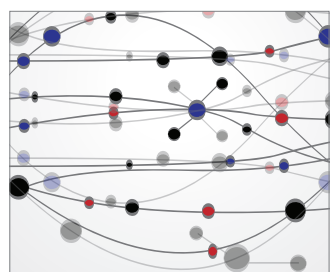

\section{The Scientific} World Journal
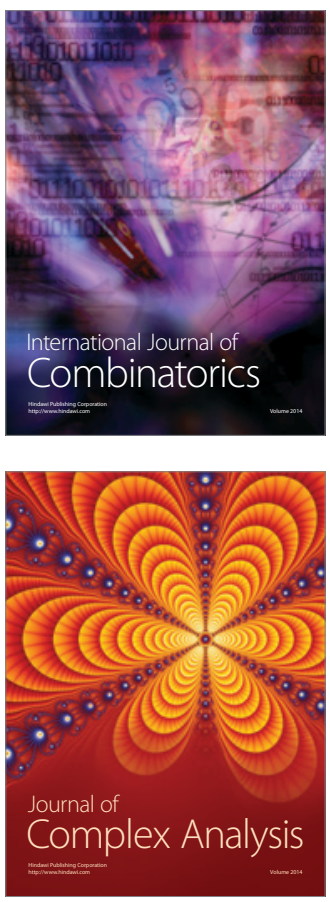

International Journal of

Mathematics and

Mathematical

Sciences
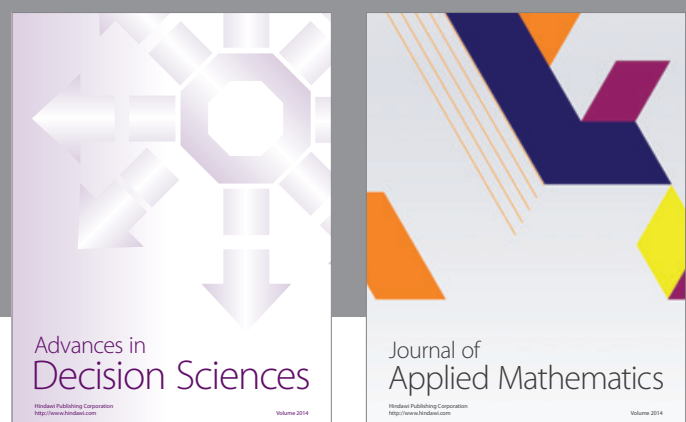

Journal of

Applied Mathematics
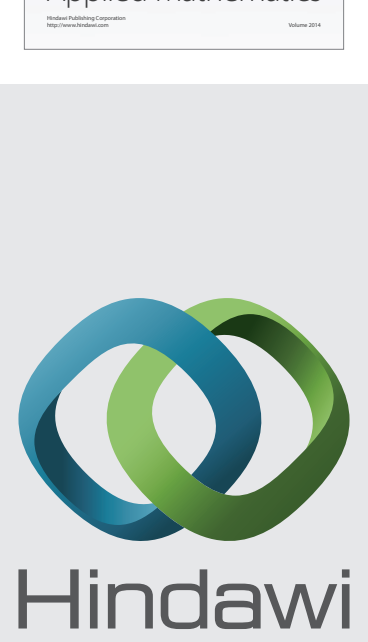

Submit your manuscripts at http://www.hindawi.com
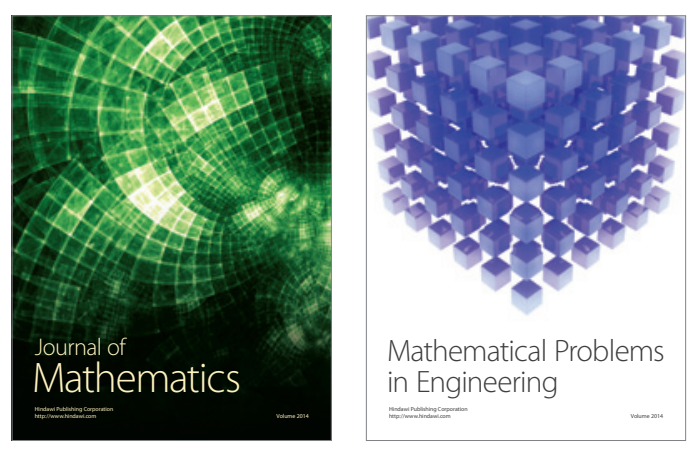

Mathematical Problems in Engineering
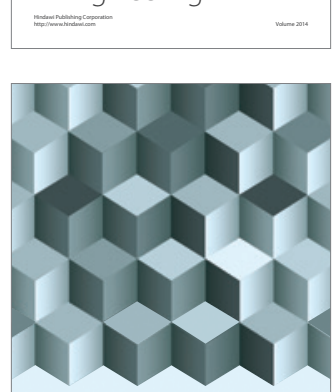

Journal of

Function Spaces
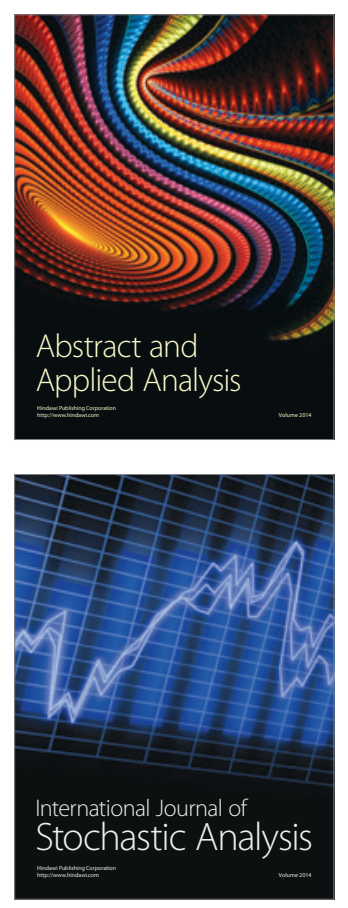

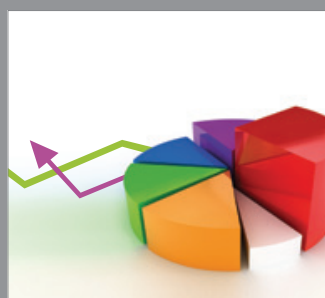

ournal of

Probability and Statistics

Promensencen
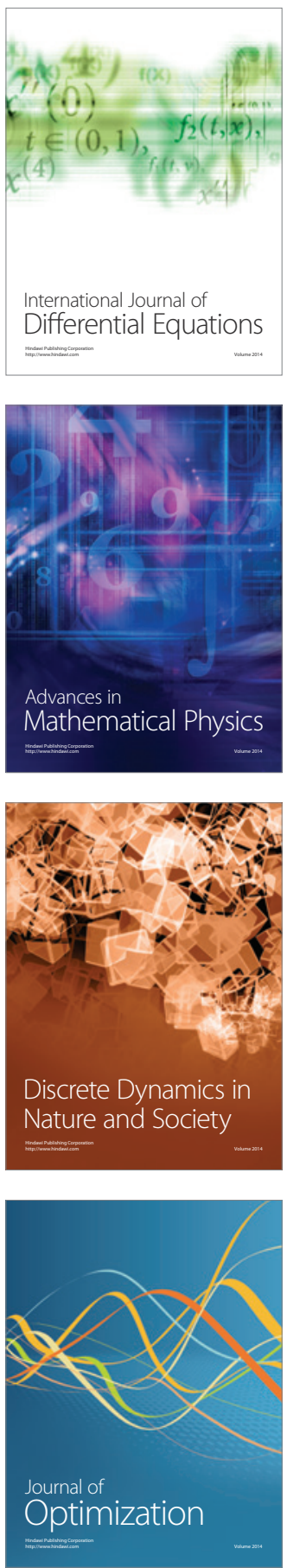